\title{
Giant neurons in the macaque pulvinar: a distinct relay subpopulation
}

\author{
Kosuke Imura ${ }^{1, a}$ and Kathleen S. Rockland ${ }^{1,2, *}$ \\ 1. Lab for Cortical Organization and Systematics, RIKEN Brain Science Institute, Japan \\ 2. Graduate School of Science and Engineering, Saitama University, Japan \\ Edited by: Javier DeFelipe, Cajal Institute (CSIC), Spain \\ Reviewed by: Marcello G. Rosa, Department of Physiology, Monash University, Australia \\ Ray W. Guillery, Department of Anatomy, School of Medicine, Marmara University, Turkey
}

\begin{abstract}
Calbindin positive $(\mathrm{CB}+)$ giant neurons are known to occur within the pulvinar nucleus in subhuman primates. Here, we demonstrate by combined retrograde tracing and immunocytochemistry that at least some of these are pulvinocortical relay neurons, and further report several distinctive features. First, in contrast with non-giant relay neurons, the giant neurons are often solitary and isolated from a main projection focus. The question thus arises of whether their cortical projections may be non-reciprocal or otherwise distinctive. Second, these neurons are positive for GluR4; but third, they are otherwise neurochemically heterogeneous, in that about one-third are positive for both parvalbumin (PV) and CB. Presumably, these subpopulations are also functionally heterogeneous. These results provide further evidence for the idea of multiple, interleaved organizations within the pulvinar; and they imply that thalamocortical projections are more disparate than has yet been appreciated. Finally, we found that giant CB+ neurons have a distinctive meshwork of large, PV+ terminations, prominent at the first dendritic branch point. In size and location, these resemble inhibitory terminations from the zona incerta or anterior pretectal nucleus (APT), as recently described in higher order thalamic nuclei in rats. One can speculate that giant neurons in the macaque pulvinar participate in a layer 5-APT-thalamus (giant neuron) extrareticular pathway, functionally distinct from the layer 6-reticular nucleus-thalamus network.
\end{abstract}

Keywords: anterior pretectal nucleus, calbindin, extrareticular, GluR4, parvalbumin, pulvinocortical relay neurons, thalamocortical

\section{INTRODUCTION}

In the subhuman primate thalamus, pulvinocortical projections originate from glutamatergic relay neurons which can be further characterized by morphological criteria as bushy or radiate (Jones, 2007; Ma et al., 1998; Sherman and Guillery, 2006), and as positive for calbindin (CB) or parvalbumin (PV). A useful distinction, strongly manifest in the ventral posterior complex, has been that PV+ neurons ("core") tend to be larger, layer 4-projecting, and topographically organized, whereas CB+ neurons ("matrix") are smaller, layer 1-projecting, and non-topographically organized (Jones and Hendry, 1989).

Interestingly, however, the $\mathrm{CB}+$ population is itself non-homogeneous, and includes a small number of scattered, giant $\mathrm{CB}+$ neurons concentrated mainly in the lateral part of the inferior pulvinar (Pli) and the lateral half of its dorsal extension ("lateral pulvinar" according to Olszewski) above the corticotectal tract (Adams etal., 2000; Cola etal., 2005; Cusick et al., 1993; Gray et al., 1999; Jones, 2007; Soares et al., 2001; Stepniewska, 2004). Although these neurons have been observed repeatedly, there is so far very little information concerning their specific proper-

* Correspondence: Kathleen S. Rockland, Lab for Cortical Organization and Systematics, RIKEN Brain Science Institute, 2-1 Hirosawa, Wako-shi, Saitama 351-0198, Japan. e-mail: rockland@brain.riken.jp

Current address:

a Department of Anatomy, School of Life Dentistry at Tokyo, The Nippon Dental University, 1-9-20 Fujimi, Chiyoda-ku, Tokyo 102-8159, Japan.

Received: 22 Aug. 2007; paper pending published: 11 Sep. 2007; accepted: 23 0ct. 2007; published online: 2 Nov. 2007

Full citation: Frontiers in Neuroanatomy (2007) 1:2 doi: 10.3389/neuro.05/002.2007 Copyright: (c) 2007 Imura, Rockland. This is an open-access article subject to an exclusive license agreement between the authors and the Frontiers Research Foundation, which permits unrestricted use, distribution, and reproduction in any medium, provided the original authors and source are credited. ties and possible function. ("The likelihood of widely differing projections of the unusually well-stained and the smaller, less well-stained CBimmunoreactive cells in the Plil [inferior pulvinar, lateral portion] is especially tantalizing and also invites investigation," Jones, 2007, p. 1069).

In this report, we provide evidence that at least some of the giant $\mathrm{CB}+$ neurons are projection neurons, and that they are neurochemically heterogeneous, with a small subpopulation positive for both CB and PV. Like neurons in the reticular nucleus (RT), the giant neurons as a group are positive for Glutamate receptor 4 (GluR4). Finally, the giant neurons commonly have proximal dendrites enmeshed with PV+ terminations. In higher order thalamic nuclei in the rat, this configuration has been associated with a specialized, extrareticular GABAergic pathway (Bokor et al., 2005).

\section{MATERIALS AND METHODS \\ Animals}

Ten macaque monkeys (Macaca mulatta and fuscata, 4.0-5.8 kg) were used for immunohistological investigations. In addition, ten other macaque monkeys ( $M$. mulatta) were available from other ongoing studies, which had tracer injections in different cortical locations (Figure 1 ) in area V4, TE0, and posterior or anterior TE (TEp, TEa). No differences were observed between the two subspecies. Tracers used were: biotinylated dextran amine (BDA, Molecular Probes, Eugene, OR; $n=3$ ); BDA and fluororuby (FR, Molecular Probes; $n=2$ ); and a newly developed adenovirus, generated by using a neuron-specific promoter synapsin 1 and an enhanced green fluorescent protein (EGFP) reporter (AdSynEGFP; Tomioka and Rockland, 2006; $n=5$ ). As terminal procedures, all animals were deeply anesthetized by an overdose of Nembutal ( $75 \mathrm{mg} / \mathrm{kg}$ i.p.), following a tranquilizing dose of ketamine (11 mg/kg i.m.), and perfused transcardially in series with: saline containing $0.5 \%$ sodium nitrite $(0.3-0.5 \mathrm{~L})$, 


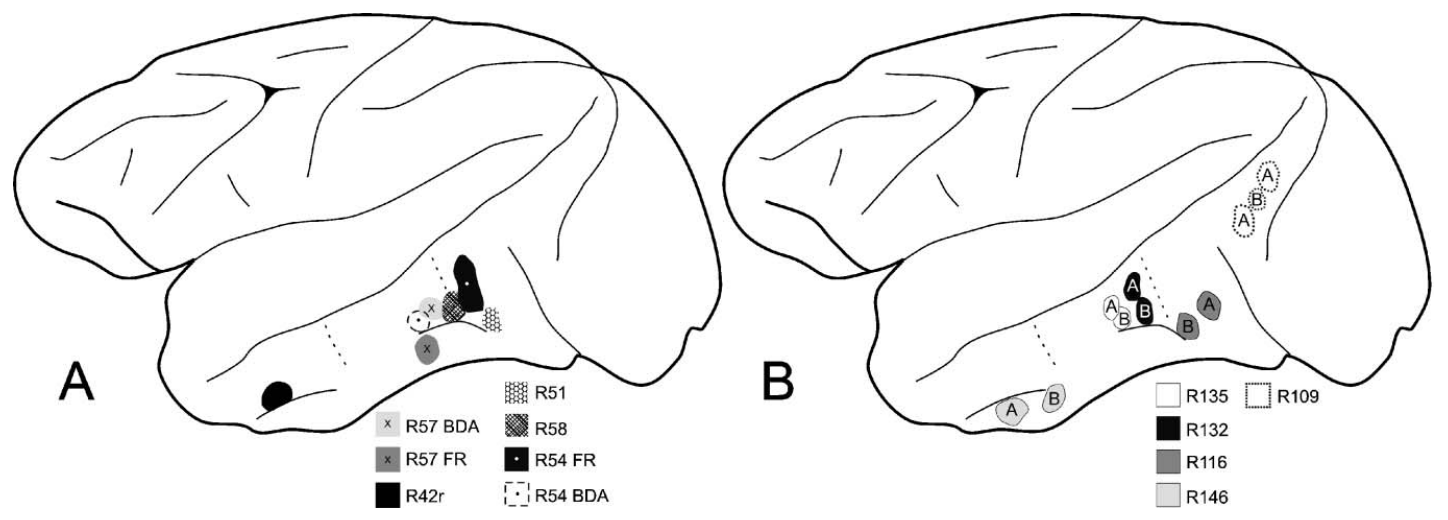

Figure 1. Schematic diagrams of the macaque left cerebral hemisphere to demonstrate location of tracer injections. (A) Five cases with injections of BDA, or BDA and FR, in anterior TE, posterior TE, or TEO. Dotted lines indicate estimated borders for these three regions (Saleem and Logothetis, 2007). (B) Five cases with injections of BDA ("B") and AdSynEGFP ("A") in anterior TE, posterior TE, TEO, or V4 (case 109).

$4 \%$ paraformaldehyde $(4 \mathrm{~L}$, in $0.1 \mathrm{M}$ phosphate buffer $[\mathrm{PB}], \mathrm{pH} 7.4)$, and $0.1 \mathrm{M}$ PB containing sucrose $(0.5 \mathrm{~L}$ each of 10,20 , and $30 \%)$. All animal procedures were carried out in conformity with official Japanese regulations for research on animals, following institutionally approved protocols (RIKEN, Brain Science Institute), and in accordance with the USA National Institutes of Health Guide for the Care and Use of Laboratory Animals (NIH Publication No. 80-23, revised 1996). Experimental protocols involving AdSynEGFP were approved by the Safety Division of the RIKEN Institute, and were carried out in biosafety level 2 rooms, also in accordance with the USA NIH Guidelines for Research Involving Recombinant DNA molecules.

\section{Surgery and tracer injections}

Surgery was carried out under sterile conditions after the animals were deeply anesthetized with Nembutal $(35 \mathrm{mg} / \mathrm{kg}$ i.p. followed by i.v. supplements as needed). Cortical areas of interest were localized by direct visualization, subsequent to craniotomy and duratomy, in relation to sulcal landmarks (i.e., superior temporal sulcus, or anterior or posterior middle temporal sulci, or, for V4 and TE0, the lunate sulcus). Two or three injections were pressure delivered through a $10 \mu \mathrm{l}$ Hamilton syringe, with a volume of $0.75-1.2 \mu \mathrm{l}$ per injection. For BDA, a $10 \%$ solution was used (1:1 mixture of 3000 and $10000 \mathrm{MW}$, in $0.0125 \mathrm{M}$ phosphate-buffered saline (PBS); pH 7.4); for FR, $10 \%$ in $0.1 \mathrm{M}$ PBS; for AdSynEGFP, $1.5 \mu \mathrm{l}$ of $1.0 \times 10^{12} \mathrm{pfu} / \mathrm{ml}$ (Tomioka and Rockland, 2006, 2007). After recovery, the animals were allowed to survive 18-22 days for axonal transport of tracers, and then perfused as above. The results among the three tracers were consistent in producing a few, scattered very large neurons in the pulvinar, but BDA produced the least complete dendritic filling.

BDA and FR have been extensively used as anterograde tracers (Veenman et al., 1992). However, in some pathways, sporadic retrograde transport is known to occur (Reiner et al., 2000). On the basis of our previous experience with these tracers, we would agree that the BDA retrograde labeling is usually subtotal ("capricious," Vercelli et al., 2000); but, while this precludes quantitative analysis, positive labeling, as long as it is understood to be subtotal, is reliable and not artifactual.

\section{Tissue preparation: tracers}

Tissue was sectioned at $50 \mu \mathrm{m}$, on a freezing microtome. Free-floating sections were processed by standard techniques for $B D A$ or $F R$, using $A B C$ kits (ABC Elite kits, Vector Laboratories, Burlingame, $C A$ ) and DAB (Zhong and Rockland, 2003). Two cases received injections of both BDA and FR. First, tissue was reacted for BDA, with the addition of $0.03 \%$ nickel ammonium sulfate to the DAB solution, in order to produce a black reaction. Next, processing was continued by the following steps: primary antibody (rabbit polyclonal anti-tetramethylrhodamine, Molecular Probes) to FR (1:6000; $4^{\circ} \mathrm{C}$ for 2 days), goat biotinylated anti-rabbit secondary antibody (1:200) and $A B C$ complex, and a final $D A B$ step, without nickel ammonium sulfate. The FR reaction product was thus distinguished as brown.

The animals with injections of AdSynEGFP also had injections of BDA As for BDA and FR, tissue was first processed for BDA (black, by nickel enhancement), and then for EGFP (brown, by DAB). The second reaction was initiated by using $1 \mu \mathrm{g} / \mathrm{ml}$ of rabbit anti-EGFP antibody (produced at RIKEN Research Resource Center (RRC)), followed by secondary antibody and $\mathrm{ABC}$ complex, and $\mathrm{DAB}$ histochemistry without nickel ammonium sulfate (Tomioka and Rockland, 2007).

\section{Single immunoperoxidase staining for GluR4 or CB}

Sections were processed from three brains for GluR4 and from one brain for $\mathrm{CB}$. These were immersed in blocking solution ( $0.1 \mathrm{M}$ PBS containing $0.5 \%$ Triton-X-100 and $5 \%$ normal goat serum), and then incubated for 2 days at $4^{\circ} \mathrm{C}$ with rabbit polyclonal anti-GluR4 (1:1000; Chemicon, Temecula, CA) or mouse monoclonal anti-CB antibody (1:1000; Swant, Bellinzona, Switzerland). Finally, sections were incubated with biotinylated anti-rabbit or antimouse secondary antibody (1:200; Vector), and visualized by $A B C$ and $D A B$.

\section{Double immunofluorescence for CB and PV; CB and GluR4}

In eight brains (including two of the four described above), 10-12 selected sections were double reacted for $\mathrm{CB}$ and $\mathrm{PV}$, or for $\mathrm{CB}$ and GluR4. For primary antibodies, we used rabbit polyclonal anti-CB antibody (1:400; Chemicon), mouse monoclonal anti-CB antibody (1:1000; Swant), mouse monoclonal anti-PV antibody (1:5000; Swant), and rabbit polyclonal anti-GluR4 antibody (1:400; Chemicon). After blocking, sections were incubated for two days at $4{ }^{\circ} \mathrm{C}$ in a mixture of two different antibodies. Then, sections were incubated for 90 minutes in the suitable combination of secondary antibodies. These were chosen from: Alexa Fluor 488conjugated anti-mouse lgG goat antibody (1:200; Molecular Probes), Alexa Fluor 594-conjugated anti-mouse IgG goat antibody (1:200; Molecular Probes), Alexa Fluor 488-conjugated anti-rabbit IgG goat antibody (1:200; Molecular Probes), Alexa Fluor 594-conjugated anti-rabbit IgG goat antibody (1:200; Molecular Probes). Sections were mounted on glass slides and coverslipped with IMMU-Mount (Thermo Shandon, Pittsburgh, PA).

\section{Double reaction for CB and BDA; CB and AdSynEGFP}

To verify directly that giant projection neurons are positive for $\mathrm{CB}$, we double reacted selected sections in one case first for $C B$ (as above, mouse monoclonal anti-CB antibody). After washing with $0.1 \mathrm{M}$ PBS, the CBreacted sections were further processed to visualize BDA by fluorescence. For this, sections were incubated for 1.5 hours at room temperature in a mixture of Alexa Fluor 488-conjugated streptavidin (st-avidin, 1:200; 

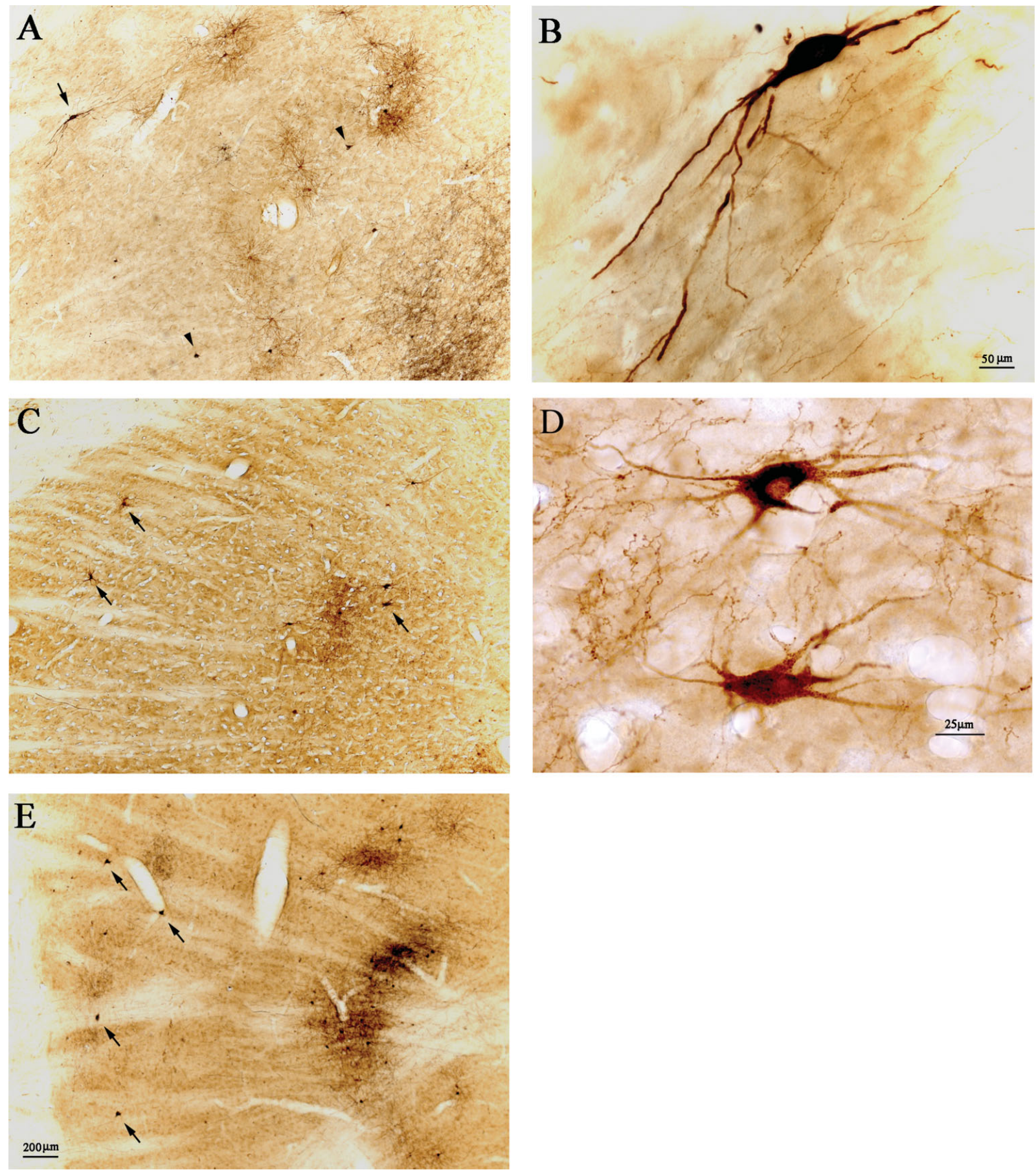

Figure 2. Retrogradely labeled pulvinocortical neurons include giant cells. (A) Injections of AdSynEGFP (brown DAB) and BDA (black, nickel-enhanced $D A B$ ) in posterior area TE (TEp) result in a dense projection focus in Plil, dorsal to the corticotectal tract. Abundant EGFP-labeled neurons (brown) are evident, with some BDA retrogradely labeled neurons (black), and abundant BDA anterogradely labeled terminations (black). Arrowheads point to two giant BDA-labeled neurons, and arrow indicates one giant EGFP-expressing neuron. Dorsal is at the top and lateral is to the left (also for $C$ and E). (B) Higher magnification of the EGFP-expressing neuron in A. (C) After injections in posterior TE, in case 57, of FR (brown DAB) and BDA (black, nickel-enhanced DAB), there is a dense projection focus. Two FR-labeled neurons at the lateral edge are indicated by arrows; and a third arrow marks another two giant neurons, located more medially, neighboring to a patch of cortical terminations. (D) Higher magnification of the two medially located neurons in C. The upper of these is double-labeled (brown FR and black BDA). (E) Injections of AdSynEGFP (brown DAB) and BDA (black, nickel-enhanced DAB) in area V4 (case 109) result in a dense projection focus (and see Figure 3). Abundant EGFP-expressing neurons (brown) are evident, with some BDA retrogradely labeled neurons (black) and abundant BDA anterogradely labeled terminations (black). Arrows indicate four relatively solitary giant BDA-labeled neurons, at the lateral edge of the pulvinar. $A, C$, and $E$ are at the same magnification. 
Molecular Probes), and secondary antibody Alexa Fluor 594-conjugated anti-mouse goat antibody (1:200; Molecular Probes).

For CB and AdSynEGFP, selected sections were incubated in a mixture of mouse monoclonal anti-CB antibody and rabbit polyclonal anti-EGFP antibody, followed by Alexa Fluor 594-conjugated anti-mouse goat antibody and Alexa Fluor 488-conjugated anti-rabbit goat antibody.

\section{Data analysis}

Sections were scanned by a Zeiss Axioskop 2 plus microscope (Carl Zeiss, Jena, Germany) using transmitted light or appropriate fluorescent filters for Alexa Fluor 488 (peak excitation, $495 \mathrm{~nm}$; peak emission, $519 \mathrm{~nm}$ ) and Alexa Fluor 594 (peak excitation, $590 \mathrm{~nm}$; peak emission, $617 \mathrm{~nm}$ ). Photographs were taken as digital images. Images were merged and adjusted for contrast and/or brightness to match the real image, by using standard image software (Adobe Photoshop 7.0, Adobe, San Jose, CA)

Soma size of $\mathrm{CB}+$ neurons was assessed by using a light microscope $(40 \times$ objective) with a camera lucida drawing apparatus ( $\mathrm{MH}-3$, Olympus, Tokyo, Japan), and tracing the long and short axis of the soma. Seven sections were used from one case reacted by DAB. Neurons $(n=50)$ were evaluated from both inferior pulvinar and its dorsal extension (classical "lateral pulvinar"). We also calculated number and density (sq. millimeter) per section in the same material.

Several nomenclatures have been introduced to designate pulvinar subdivisions, mainly based on neurochemical criteria (Adams et al., 2000; Gutierrez et al., 1995; Stepniewska, 2004; and summarized in Jones, 2007). A standardization suggested by Jones uses "lateral" and "inferior pulvinar," but with borders modified from the classical Olszewski subdivisions. Notably, the ventral portion of the lateral pulvinar is considered an extension of Pli. We have followed this subdivision and designate this portion as Plil.

\section{RESULTS}

\section{Distribution}

Consistent with several previous studies, immunohistochemistry for $\mathrm{CB}$ revealed a small number of giant neurons conspicuous for their large soma size and intense CB staining. Average soma size $(n=50)$ was ascertained as 20 (short axis) $\times 30 \mu \mathrm{m}$ (long axis), with $42.5 \mu \mathrm{m}$ being the longest axis. As illustrated by others, this is about twice the size of small $\mathrm{CB}+$ neurons (Figure 4D in Jones and Hendry, 1989; Figure 7 in Cola et al., 2005). These neurons tended to be solitary, with a spacing of 300-500 $\mu \mathrm{m}$. They are concentrated laterally, above and below the corticotectal tract (Cola et al., 2005; Cusick et al., 1993; Soares et al., 2001; and see distribution map in Figure 10.10, Jones, 2007).

\section{Tracer injections}

To assess whether giant $\mathrm{CB}+$ neurons project to cortical areas, we first scanned histology slides from brains with tracer injections in different cortical regions. Injections were in area V4 and several temporal subdivisions, extending from area TEO to anterior dorsal TE (Figure 1).

In all these cases, a small number of very large retrogradely filled neurons could be discerned, in addition to a larger number of smaller neurons (Figures 2 and 3). The large neurons were spaced about 300-700 $\mu \mathrm{m}$ apart and varied from $0-6$ per section. Scoring three sequential sections in case 109 (Figure 3), we counted four giant neurons (BDA-labeled) and 42 non-giant (18 labeled by BDA and 24 by AdSynEGFP), three giant neurons (two labeled by BDA and one by AdSynEGFP) and 56 non-giant (28 labeled by each tracer), nine giant neurons (six labeled by BDA and three by AdSynEGFP; one double-labeled) and 68 non-giant (47 labeled by BDA and 21 by AdSynEGFP). Giant neurons tended to occur preferentially ventral and lateral in Plil or, to a lesser extent, in Pli below the corticotectal tract, in its lateral portion. Often, these did not overlap with the densest part of the main projection focus, but were offset slightly more than $1.0 \mathrm{~mm}$ laterally from this (Figures 2 and 3 ).

One hallmark of giant $\mathrm{CB}+$ neurons is that they have radiating dendrites (Jones, 2007). In our material, only limited, proximal dendritic filling
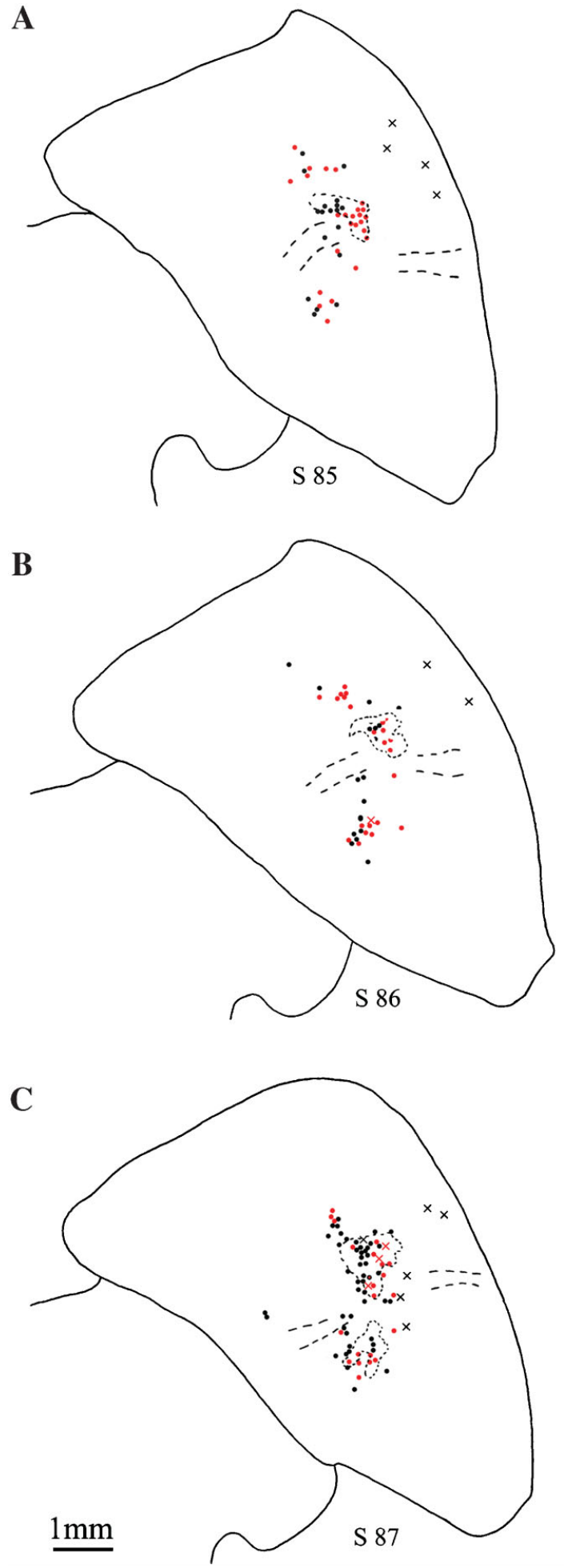

Figure 3. Distribution of pulvinocortical neurons. (A-C) Line drawings of the pulvinar from three sequential sections (s 85, 86, 87; case 109), showing the distribution of retrogradely labeled pulvinocortical neurons. Giant neurons $(X)$ are few, usually isolated, and are often located laterally. Non-giant neurons are indicated by filled circles (1 circle or $X=1$ neuron). A few of these occur away from the dense projection focus. BDA-labeled neurons are in black; AdSynEGFP-labeled neurons, in red; and concentrations of dense anterograde label (BDA only) are shown by dotted lines. Dashed lines indicate approximate location of corticotectal tract. Medial is to the left. 
was produced by BDA. AdSynEGFP resulted in more extensive, Golgi-like filling, but only a small number of giant neurons occurred after these injections. For one obviously giant neuron, dendritic spread was measured as $360 \mu \mathrm{m}$ and $680 \mu \mathrm{m}$ from the soma (Figure 2B). For another neuron (not illustrated), the dendritic tree was more spherical, with a radius of $400 \mu \mathrm{m}$. This contrasts with the smaller tree of "bushy" pulvinocortical neurons, where the dendritic arbor is about $300 \mu \mathrm{m}$ in diameter.

The most abundant labeling of giant neurons resulted from the BDA injection in posterior area TE (case 57); but, given the "capricious" nature of FR and BDA as retrograde tracers, it is very likely that not all the pulvinocortical projection neurons have been labeled. That is, even in case 57 , the number of giant neurons is much less than what is evident after CB immuno-labeling.

In order to ascertain more directly that large $\mathrm{CB}+$ neurons were in fact projection neurons, we double-reacted selected sections by immunofluorescence for antibodies against CB and EGFP ( $n=2$ brains), or for anti-CB combined with streptavidin conjugated with Alexa 594, to visualize BDA ( $n=1$ brain). For eleven neurons, we were able to verify directly that giant retrogradely labeled neurons are $\mathrm{CB}+$ (Figure 4). None of the giant projection neurons were $\mathrm{CB}-$, but we stress that screening was limited to a few sections.

\section{Neurochemical properties}

Double immuno-labeling for CB and PV demonstrated two subpopulations, in an approximate ratio of $2: 1$. That is, most of the giant neurons were only $\mathrm{CB}+(n=88)$, but about one-third $(n=39)$ were both $\mathrm{CB}+$ and PV+ (Figure 5). Giant neurons positive for GluR4 occur in about the same density and location as giant $\mathrm{CB}+$ neurons (Figure 6). Double immunolabeling for $\mathrm{CB}$ and GluR4 directly showed that all giant $\mathrm{CB}+$ neurons were also positive for GluR4 (Figure 7).

In the material double reacted for $\mathrm{CB}$ and $\mathrm{PV}$, we noticed that the proximal dendritic portions of giant $\mathrm{CB}+$ neurons were commonly enmeshed with large $\mathrm{PV}+$ terminations. These were particularly obvious beyond the first dendritic branch point (Figure 8). In DAB-reacted material, a similar configuration of PV + terminations could be discerned in association with proximal dendrites of very large PV+ neurons (not illustrated).

\section{DISCUSSION}

The population of giant $\mathrm{CB}+$ neurons has been previously noted by several investigators (Adams et al., 2000; Cola et al., 2005; Cusick et al., 1993; Gray et al., 1999; Jones, 2007; Soares et al., 2001; Stepniewska, 2004); and their distribution within the pulvinar has recently been mapped (Figure 10.10 in Jones, 2007). In this study, we have reported three further, functionally relevant observations, as discussed below.

First, we have verified that at least some giant neurons are pulvinocortical relay cells. This is shown indirectly, based on soma size and location after retrograde tracer injections in cortical areas. More directly, giant retrogradely labeled neurons are double-labeled for $\mathrm{CB}$. We note that the number of very large, retrogradely filled neurons was markedly less than the number of giant $\mathrm{CB}+$ neurons (see our Figure 6 (GluR4), and Figure 10.10 in Jones, 2007). This is probably because the BDA and FR are only sporadic retrograde tracers; but the AdSynEGFP injections also revealed only a few very large neurons. The possibility remains that some of these neurons are not projection neurons, or that the cortical target is different from regions we injected. Double labeling for CB and a conventional retrograde tracer, such as cholera toxin, could clarify this issue.

The giant relay cells tend to be solitary, not clustered. The more numerous, non-giant pulvinocortical relay neurons are characteristically clustered together, although a few outliers can usually be detected. In our material, the very large neurons are usually offset about $1.0 \mathrm{~mm}$ laterally from the main projection focus. In the main projection focus, anterograde and retrograde labeling is dense and overlapping. There is frequently no anterograde labeling at all in the immediate vicinity of the giant neurons, or only sparse labeling. Our interpretation is that these neurons may have preferentially one-way, non-reciprocal connections with their cortical tar-
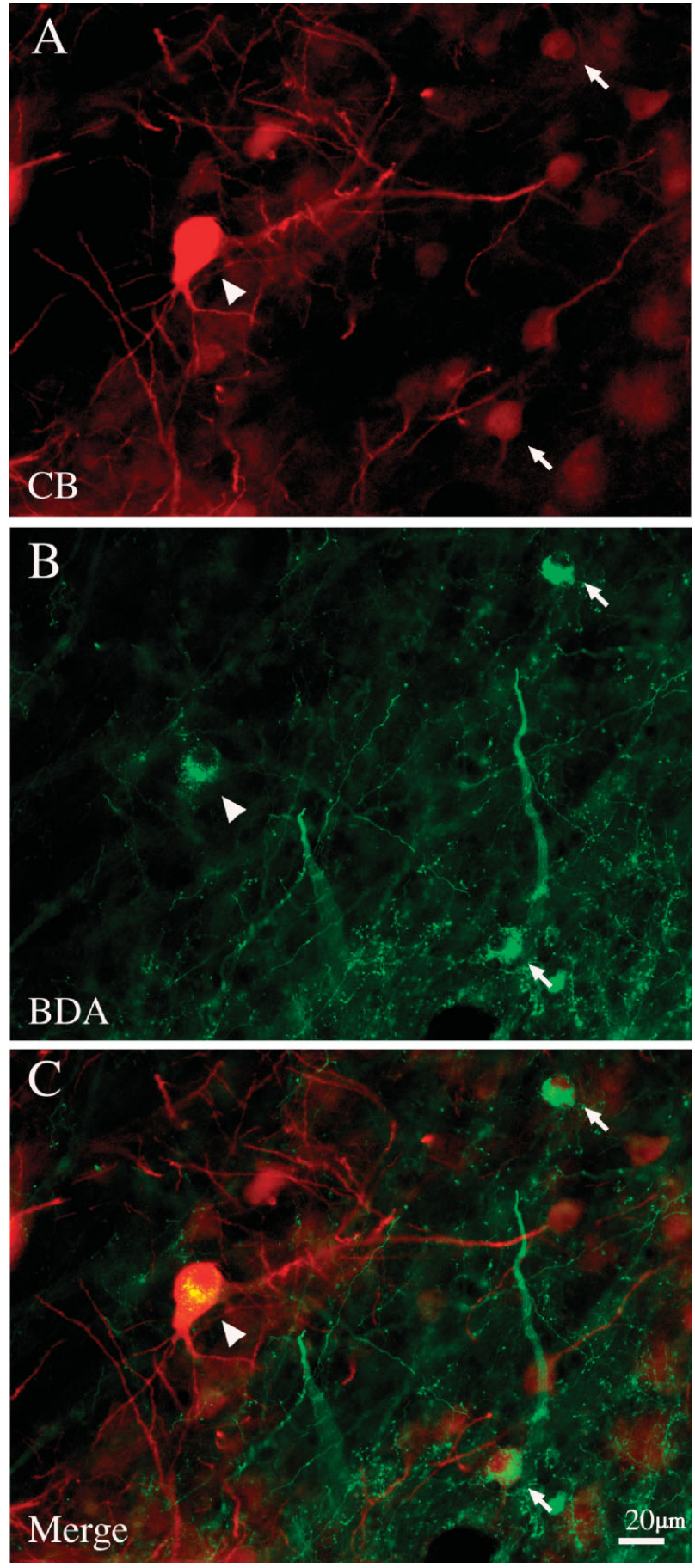

Figure 4. Giant pulvinocortical relay neurons retrogradely labeled by BDA are $\mathbf{C B}+$. (A) Giant neuron (arrowhead) intensely immunofluorescent for CB. Two other, smaller $C B+$ neurons are indicated by arrows. (B) Same section, reacted with streptavidin-Alexa488 to visualize BDA transported from an injection in posterior area TE. Arrowhead and arrows indicate the same three neurons as in $A$. (C) Merged image directly demonstrates that these three $C B+$ neurons are also labeled by BDA.

gets. At least, these may be considerably less dense than for non-giant neurons. Alternately, they may receive cortical terminations, but not from their own cortical targets.

A few giant projection neurons were double-labeled by injections of BDA and FR, which were separated by $5.0 \mathrm{~mm}$ in dorsal and ventral por- 

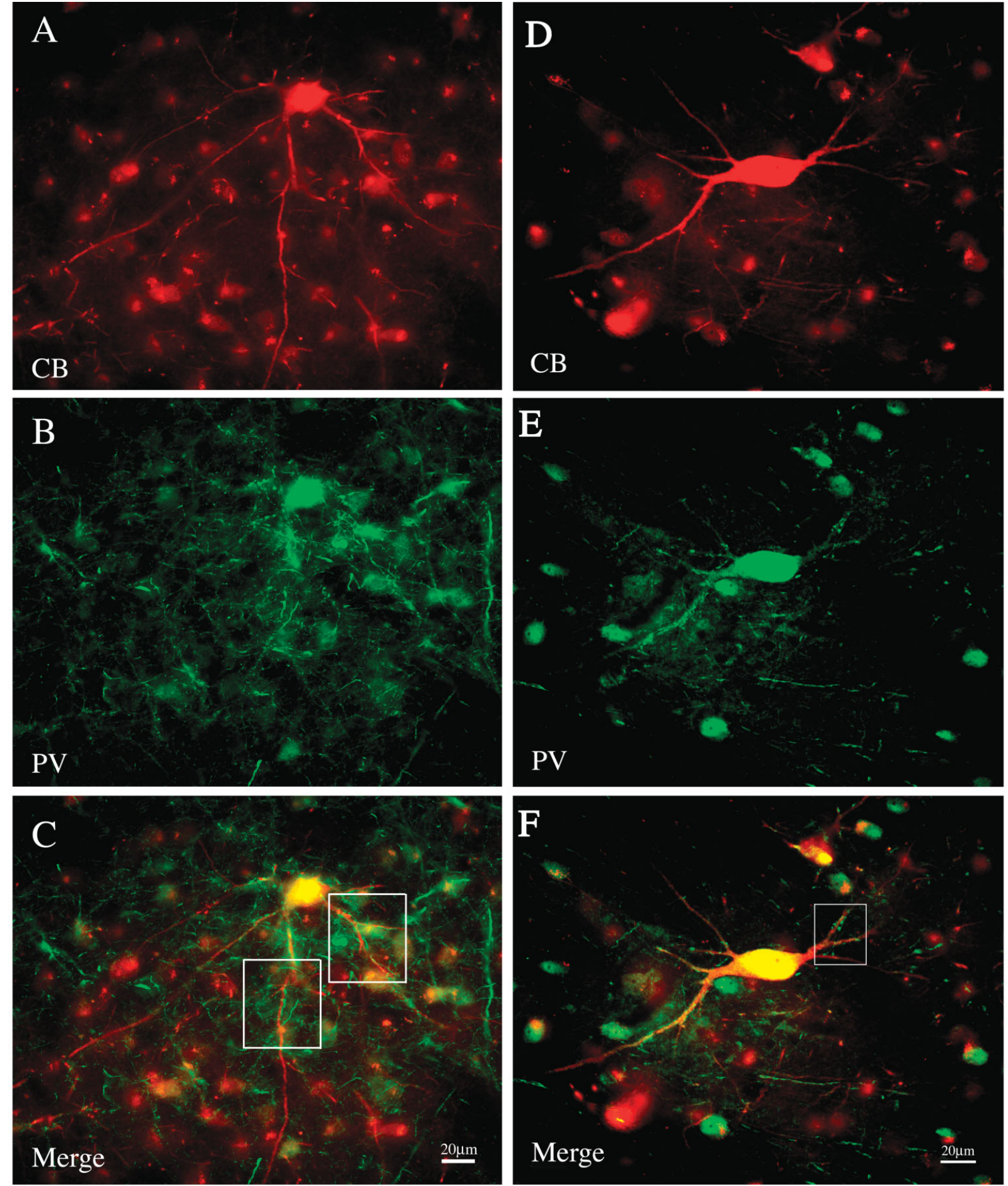

Figure 5. A subset of giant neurons is positive for both CB and PV. (A) A field of $C B+$ neurons from PI, including one giant neuron (immunofluorescense for Alexa-594). (B) Same section reacted for PV (immunofluorescense for Alexa 488). (C) Merged image directly demonstrates that the giant neuron is both CB+ and $P V+$. Boxed segments are shown at higher magnification in Figure 8. (D) Giant CB+ neuron from another animal. (E) Same section reacted for PV. (F) Merged image directly demonstrates that the giant neuron is both $C B+$ and $P V+$. Boxed segment is shown at higher magnification in Figure 8.

tions of area TEp (Figure 1). This could mean that, like the smaller, matrix $\mathrm{CB}$ neurons in the somatosensory thalamus, these giant neurons tend to have diffuse, cross-area projections. However, more experiments, using more widely spaced injections, will be necessary to determine this issue. As it is, the distinction of layer 1-versus layer 4-projecting pulvinocortical neurons may be less sharp in the occipitotemporal region; single axons in this region can arborize in both layer 1 and the middle lay- ers (Rockland etal., 1999). Some of the giant neurons, as noted, are positive for both CB and PV (see also Jones and Hendry, 1989 about $\mathrm{CB}+\mathrm{PV}+$ neurons).

A second significant feature is that these giant neurons in their proximal dendritic portions, beyond the first branch point, are enmeshed with PV+ large terminations. Among the most likely sources of these terminations are the RT, zona incerta, or the anterior pretectal nucleus (APT), all of which

6

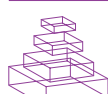

Frontiers in Neuroanatomy | November 2007 | Volume 1 | Article 2 


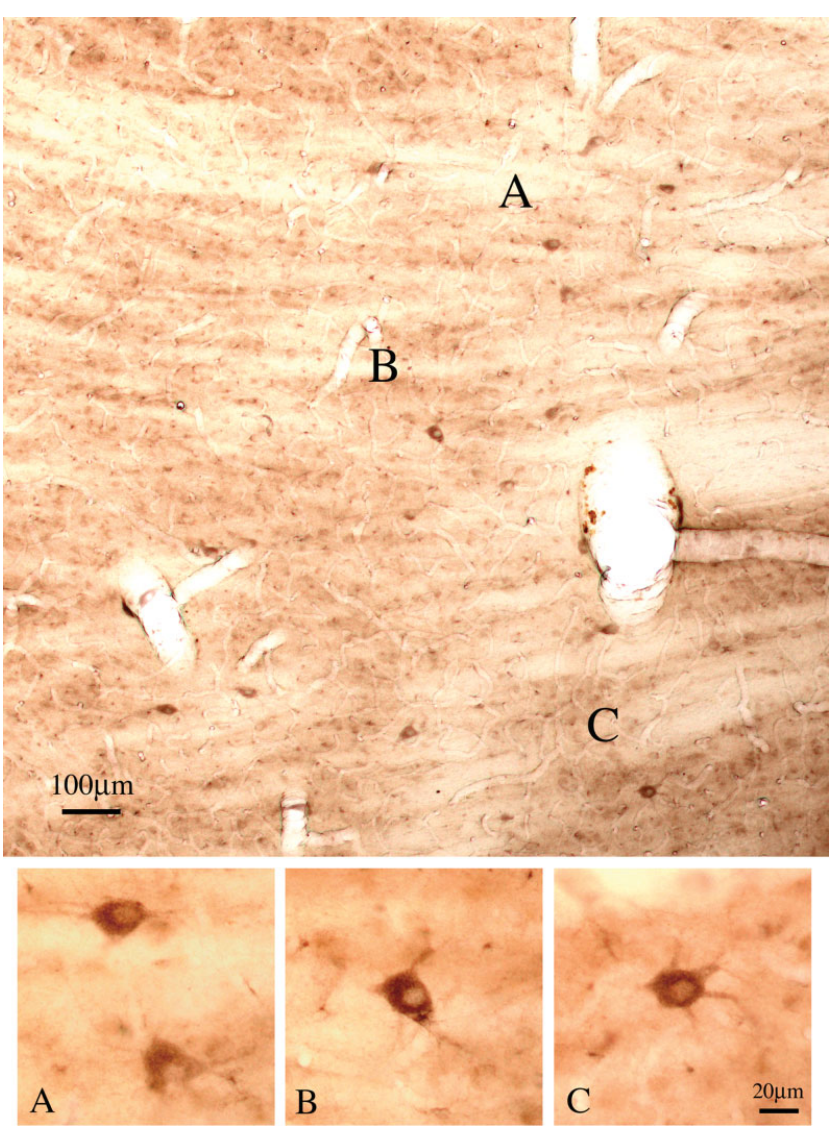

Figure 6. Giant neurons are positive for GluR4. Coronal section overview through Plil (lateral is to the left), with scattered large neurons that are strongly positive for GluR4. (A-C) Four neurons, at higher magnification.

have GABAergic terminations (Bartho et al., 2002). The proximal location may argue against $\mathrm{RT}$, which tends to target more distal dendritic locations (llinsky et al., 1999; Liu et al., 1995); and the large size rules out a fourth possible source, from recently described PV+ GABAergic interneurons (Imura and Rockland, 2006). Thus, we suggest that these giant neurons participate in what is described in higher order nuclei of rats as a distinct extrareticular pathway. In rats, this exerts a powerful GABAergic control, with multiple release sites and $\mathrm{GABA}_{\mathrm{A}}$-mediated responses, appropriate for fast, phasic control (Bokor et al., 2005). In the cat, as well, there are dense pretectal terminals to the pulvinar nucleus, but these are both nonGABAergic (87.6\%) and GABAergic (12.4\%). They are reported to contact mainly CB - pulvinocortical relay (Baldauf et al., 2005), although the cat pulvinar contains some very large CB+ neurons (Batini et al., 1991; and personal communication, Hisayuki Ojima).

Given evident species differences, it remains uncertain whether, as in the rat, the giant $\mathrm{CB}+$ neurons in the monkey constitute a distinct, extrareticular subsystem. An interesting further possibility, however, would be that there is a layer 5-APT-thalamus (giant neuron) pathway, operating in parallel with a layer 6-RT-thalamus pathway, as suggested by Bokor et al. Type 2 ("round") corticopulvinar axons have been shown to project by collaterals to both the pretectum and pulvinar (Figure 7 in Rockland, 1998), and it will evidently be important to further investigate this circuitry and its functional significance.

Third, we show that the giant neurons are all positive for GluR4, and in this respect, they are similar to RT neurons. In addition, however, about one-third are co-labeled with PV. From this, one can infer that there are further subpopulations even within this group of giant neurons. In terms
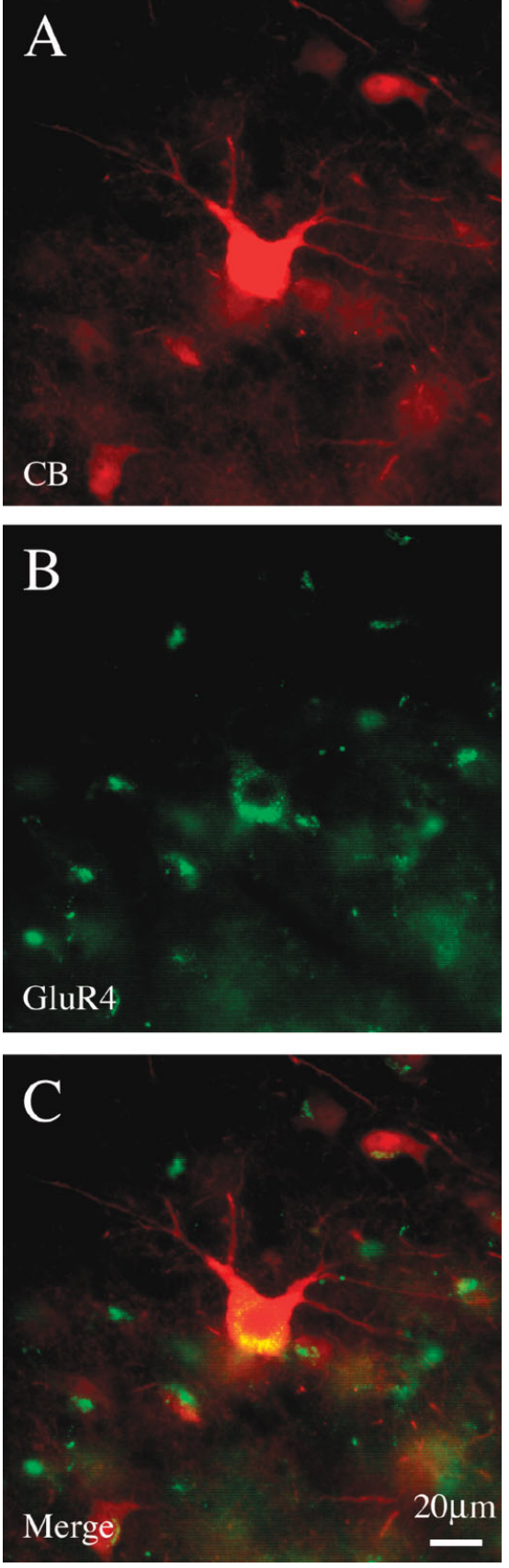

Figure 7. Giant $\mathbf{C B}+$ neurons are co-labeled by GluR4. (A) Giant $C B+$ neuron. (B) Same section, reacted for GluR4. (C) Merged image directly demonstrates co-labeling of CB and GluR4. (Small cells are glia, which are positive for GluR4.)

of subpopulations, some investigators have distinguished two subtypes of type 1 corticopulvinar axons and four subtypes of type 2 (Kultas-llinsky et al., 2003). If pulvinocortical, as well as corticopulvinar projections prove to be more diverse than presently recognized, this would have obvious consequences for our understanding of modes of communication between cortex and thalamus.

\section{CONFLICT OF INTEREST STATEMENT}

The authors declare that the research was conducted in the absence of any commercial or financial relationships that could be construed as a potential conflict of interest. 

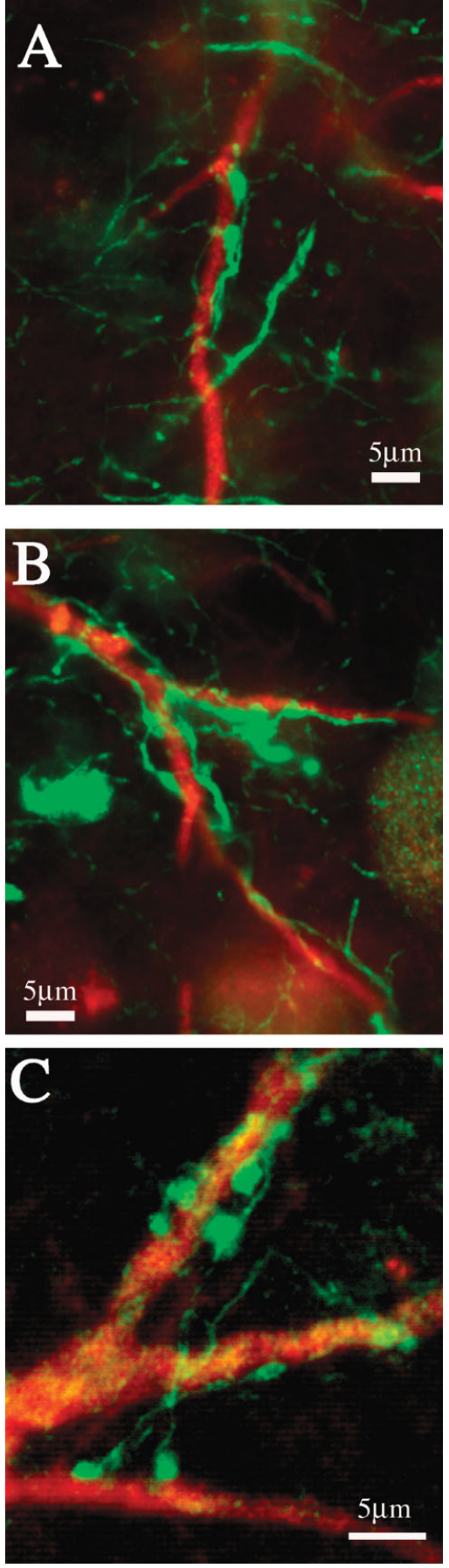

Figure 8. (A-C) Proximal dendrites of giant neurons are densely coated with PV+ terminations. Higher magnification from the three boxed regions in Figures $\mathbf{5 C}$ and $\mathbf{5 F}$.

\section{ACKNOWLEDGEMENTS}

We thank Elena Borra for assistance with Figure 1, Hiromi Mashiko and Yoshiko Abe for expert assistance with histology, Michiko Fujisawa for assistance with manuscript preparation, and members of the Lab for Cortical Organization and Systematics for discussion and general cooperation. This work was supported by research funding from RIKEN Brain Science Institute.

\section{REFERENCES}

Adams, M. M., Hof, P. R., Gattass, R., Webster, M. J., and Ungerleider, L. G. (2000). Visual cortical projections and chemoarchitecture of macaque monkey pulvinar. J. Comp. Neurol. 419, 377-393.

Baldauf, Z. B., Wang, S., Chomsung, R. D., May, P. J., and Bickford, M. E. (2005). Ultrastructural analysis of projections to the pulvinar nucleus of the cat. II: Pretectum. J. Comp. Neurol. 485, 108-126.

Bartho, P., Freund, T. F., and Acsady, L. (2002). Selective GABAergic innervation of thalamic nuclei from zona incerta. Eur. J. Neurosci. 16, 999-1014.

Batini, C., Guegan, M., Palestini, M., and Thomasset, M. (1991). The immunocytochemical distribution of calbindin-D28k and parvalbumin in identified neurons of the pulvinarlateralis posterior complex of the cat. Neurosci. Lett. 130, 203-207.

Bokor, H., Frere, S. G., Eyre, M. D., Slezia, A., Ulbert, I., Luthi, A., and Acsady, L. (2005). Selective GABAergic control of higher-order thalamic relays. Neuron 45, 929-940.

Cola, M. G., Seltzer, B., Preuss, T. M., and Cusick, C. G. (2005). Neurochemical organization of chimpanzee inferior pulvinar complex. J. Comp. Neurol. 484, 299-312.

Cusick, C. G., Scripter, J. L., Darensbourg, J. G., and Weber, J. T. (1993). Chemoarchitectonic subdivisions of the visual pulvinar in monkeys and their connectional relations with the middle temporal and rostral dorsolateral visual areas, MT and DLr. J. Comp. Neurol. 336, 1-30.

Gray, D., Gutierrez, C., and Cusick, C. G. (1999). Neurochemical organization of inferior pulvinar complex in squirrel monkeys and macaques revealed by acetylcholinesterase histochemistry, calbindin and Cat-301 immunostaining, and Wisteria floribunda agglutinin binding. J. Comp. Neurol. 409, 452-468.

Gutierrez, C., Yaun, A., and Cusick, C. G. (1995). Neurochemical subdivisions of the inferior pulvinar in macaque monkeys. J. Comp. Neurol. 363, 545-562.

Ilinsky, I. A., Ambarderkar, A. V., and Kultas-Llinsky, K. (1999). Organization of projections from the anterior pole of the nucleus reticularis thalami (NRT) to subdivisions of the motor thalamus: light and electron microscopic studies in the rhesus monkey. J. Comp. Neurol. 409, 369-384.

Imura, K., and Rockland, K. S. (2006). Long-range interneurons within the medial pulvinar nucleus of macaque monkeys. J. Comp. Neurol. 498, 649-666.

Jones, E. G. (2007). The Thalamus (Cambridge, University Press).

Jones, E. G., and Hendry, S. H. (1989). Differential calcium binding protein immunoreactivity distinguishes classes of relay neurons in monkey thalamic nuclei. Eur. J. Neurosci. 1, 222-246.

Kultas-llinsky, K., Sivan-Loukianova, E., and Ilinsky, I. A. (2003). Reevaluation of the primary motor cortex connections with the thalamus in primates. J. Comp. Neurol. $457,133-158$

Liu, X. B., Warren, R. A., and Jones, E. G. (1995). Synaptic distribution of afferents from reticular nucleus in ventroposterior nucleus of cat thalamus. J. Comp. Neurol. 352, 187-202.

Ma, T. P., Lynch, J. C., Donahoe, D. K., Attallah, H., and Rafols, J. A. (1998). Organization of the medial pulvinar nucleus in the macaque. Anat. Rec. 250, 220-237.

Reiner, A., Veeman, C. L., Medina, L., Jiao, Y., Ma, N. D., and Honig, M. G. (2000). Pathway tracing using biotinylated dextran amines. J. Neurosci. Methods 103, 23-37.

Rockland, K. S. (1998). Convergence and branching patterns of round, type 2 corticopulvinar axons. J. Comp. Neurol. 390, 515-536.

Rockland, K. S., Andresen, J., Cowie, R. J., and Robinson, D. L. (1999). Single axon analysis of pulvinocortical connections to several visual areas in the macaque. J. Comp. Neurol. 406, 221-250.

Saleem, K. S., and Logothetis, N. K. (2007). A combined MRI and histology atlas of the rhesus monkey brain in stereotaxic coordinates (Amsterdam, Elsevier).

Sherman, S. M., Guillery, R. W. (2006). Exploring the thalamus and its role in cortical function (Cambridge, MIT Press).

Soares, J. G., Gattass, R., Souza, A. P., Rosa, M. G., Fiorani, M. Jr. and Brandao, B. L. (2001). Connectional and neurochemical subdivisions of the pulvinar in Cebus monkeys. Vis. Neurosci. 18, 25-41.

Stepniewska, I. (2004). The pulvinar complex. In The Primate Visual System, J. H. Kaas, C. E. Collins, eds. (Boca Raton, CRC Press), pp. 53-80.

Tomioka, R., and Rockland, K. S. (2006). Improved Golgi-like visualization in retrogradely projecting neurons after EGFP-adenovirus infection in adult rat and monkey. J. Histochem. Cytochem. 54, 539-548.

Tomioka, R., and Rockland, K. S. (2007). Long-distance corticocortical GaBAgeric neurons in the adult monkey white and gray matter. J. Comp. Neurol. 505, 526-538.

Veenman, C. L., Reiner, A., and Honig, M. G. (1992). Biotinylated dextran amine as an anterograde tracer for single- and double-labeling studies. J. Neurosci. Methods 41, 239-254.

Vercelli, A., Repici, M., Garbossa, D., and Grimaldi, A. (2000). Recent techniques for tracing pathways in the central nervous system of developing and adult mammals. Brain Res. Bull. 51, 11-28.

Zhong, Y. M., and Rockland, K. S. (2003). Inferior parietal lobule projections to anterior inferotemporal cortex (area TE) in macaque monkey. Cereb. Cortex. 13, 527-540. 